\title{
Electrostatic Forces Control the Negative Allosteric Regulation in a Disordered Protein Switch
}

Yanming Wang ${ }^{1}$ and Charles L. Brooks III ${ }^{1,2^{*}}$

1. Department of Chemistry and 2. Biophysics Program, University of Michigan, Ann Arbor, Michigan 48109, United States

Corresponding Author

*Correspondence to: brookscl@umich.edu 


\section{Supporting Information}

\section{SI Text}

The Coarse-grained Model. We used a native centric electrostatics inclusive (EI) Gō-like model originally developed by Karanicolas and Brooks ${ }^{1}$ (EIKB model) to study the negative allosteric effect in the TAZ1 protein switch. This model was originally designed to study protein folding mechanisms $^{2}$, and was recently adopted to study protein-protein interactions ${ }^{3,4}$. Notably, the EIKB model has successfully captured the positive allosteric effect in the KIX domain of the CBP/P300 transcription co-activator. ${ }^{4}$ In this model, each amino acid residue is represented as a single bead with mass equal to the corresponding amino acid, centered at the $\mathrm{C} \alpha$ position, and connected to neighboring residues via virtual bonds. The EIKB model mainly considers two types of interactions, bonded and non-bonded interactions (equations 1-2). The bonded interactions include bond, angle, and dihedral terms. The bond and angle terms are harmonic potentials with equilibrium values set to those calculated from the native structure. The dihedral term is a statistical potential based on probability distributions obtained from the Ramachandran plot and provides additional sequence-specific information to avoid overfitting to the native structure. ${ }^{1}$ The nonbonded interactions consider the electrostatic interactions, native contacts, and the non-native excluded volume interactions. The electrostatic interactions were represented by a simple DebyeHückel potential with two parameters: dielectric constant D and screening length $\kappa$. This simple representation of electrostatics has been shown to be compatible with the coarse-grained model. ${ }^{5}$ The native contacts formed between residue pairs are modeled using a modified 12-10-6 LennardJones potential with a desolvation penalty. The interaction strength is proportional to the statistical potential $(\mathrm{i}, \mathrm{j})$ of the Miyazawa-Jernigan matrix. ${ }^{6}$ 


$$
\begin{aligned}
& U_{\text {bonded }}=\sum_{\text {bonds }}^{N-1} K_{b}\left(r_{i}-r_{0}\right)^{2}+\sum_{\text {angles }}^{N-2} K_{\theta}\left(\theta_{i}-\theta_{0}\right)^{2}+\sum_{\text {dihedrals }}^{N-3} \sum_{n}^{4} K_{\phi}\left(1+\cos \left(n \theta-\theta_{0}\right)\right) \\
& U_{\text {unbonded }}=\sum_{i>j} \frac{332 q_{1} q_{2}}{D r_{i j}} e^{-\frac{r_{i j}}{\kappa}}+\sum_{\substack{\text { native } \\
|i-j|>3}} \varepsilon\left\{13\left(\frac{\sigma_{i j}}{r_{i j}}\right)^{12}-18\left(\frac{\sigma_{i j}}{r_{i j}}\right)^{10}+4\left(\frac{\sigma_{i j}}{r_{i j}}\right)^{6}\right\}+\sum_{\substack{\text { non-native } \\
|i-j|>3}} \varepsilon\left(\frac{\sigma_{i j}}{r_{i j}}\right)^{12}
\end{aligned}
$$

The PDB structures of binary complexes TAZ1:HIF-1 $\alpha$ (1L8C) and TAZ1:CITED2 (1R8U) were used to construct the intra-molecular force-field terms of the two peptides HIF-1 $\alpha$ and CITED2 and the inter-molecular force-field terms between the two peptides and TAZ1. The PDB structure of the free TAZ1 $(1 \mathrm{U} 2 \mathrm{~N})$ was used to construct the intra-molecular terms of TAZ1 to avoid potential biases. Notably, TAZ1 has three zinc fingers with the zinc atom binding to three adjacent cysteine residues and a histidine residue $(\mathrm{Zn}-\mathrm{CCCH})$. Since no zinc finger force-field was ever built for the EIKB coarse-grained model, we chose the Zinc AMBER Force Field ${ }^{7}$ (ZAFF) as a reference and used a single bead to represent each zinc finger. Each coarse-grained zinc finger bead has a net charge of -1 calculated from the ZAFF charge distributions. A harmonic bond potential with an empirical force constant of $50 \mathrm{kcal} / \mathrm{mol}$ is used for the four $\mathrm{Zn}$-residue bonds of each zinc finger bead. A harmonic angle potential with an empirical force constant of $30 \mathrm{kcal} / \mathrm{mol}$ is used for the residue1-Zn-residue2 angle force field of each zinc finger bead.

The Calibration of the Coarse-grained Model. Previous studies have shown the EIKB Gō model tends to overestimate the intrinsic helicities of intrinsically disordered proteins (IDPs) while underestimating the strengths of IDP-protein interactions. To address these two issues, we adopted an approach similar to previous studies., ${ }^{3,4}$ Two scaling factors $\alpha$ and $\beta$ were used to scale the intraand inter-molecular interactions (equation 3), so that the model recapitulates some fundamental experimental results. The scaling factor $\alpha$ tunes the intrinsic helicities of HIF-1 $\alpha$ and CITED2 and was added to scale all intra-molecular native contacts of the two peptides. Experiments show the 
unbound HIF-1 $\alpha$ and unbound CITED2 are highly disordered. ${ }^{8,9}$ We empirically set the values of $\alpha$ to be 0.05 for both peptides. The scaling factor $\beta$ tunes the inter-molecular native contacts between TAZ1 and the two peptides so that the modeled peptides in the two binary complexes TAZ1:CITED2 and TAZ1:HIF-1 $\alpha$ reproduce the experimental dissociation constants $\left(\mathrm{K}_{\mathrm{d}}=10 \mathrm{nM}\right.$ for both peptides $\left.{ }^{10}\right)$.

$$
U_{\text {native }}=\alpha U_{\text {intra }}+\beta U_{\text {inter }}
$$

Enhanced Sampling Molecular Dynamics Simulation Protocol. We used an enhanced sampling method based on Hamiltonian replica exchange (HREX) that has shown success in modeling the positive allosteric effect in the KIX domain of $\mathrm{CBP} / \mathrm{P} 300 .{ }^{11}$ This method uses two variables $\beta$ and the temperature (T) in the Hamiltonian as the two exchange variables. The values of $\beta$ for HIF-1 $\alpha$ $\left(\beta_{\text {TAZ1:HIF-1 } \alpha}\right)$ were chosen to be span the range from 1.40 to 1.60 with an increment of 0.02 . The values of $\beta$ for CITED2 ( $\beta_{\text {TAZ1:CITED2 }}$ ) were chosen to be span the range from 1.10 to 1.30 with an increment of 0.02 . The values of $\mathrm{T}$ were set to be $300 \mathrm{~K}, 320 \mathrm{~K}$ and $340 \mathrm{~K}$. Therefore, each HREX simulation has $11 \beta$ windows and 3 temperature windows with a total of 33 combined simulation windows. Only the trajectories at $300 \mathrm{~K}$ were used for the data analysis. The cartesian coordinates of the two nearest windows $\left(T_{i}, \beta_{i}\right)$ and $\left(T_{i+1}, \beta_{i+1}\right)$ were exchanged every 10,000 steps using the Metropolis algorithm. ${ }^{12}$ All molecular dynamics (MD) simulations were carried out using the OpenMM library. ${ }^{13}$ The HREX method was implemented using the OpenMM C++ API and Message Passing Interface (MPI). All MD simulations used the Langevin integrator with a friction coefficient of $0.1 \mathrm{ps}^{-1}$ and a timestep of 22 fs to propagate the equations of motion. Periodic boundary conditions with a $150 \AA$ cubic box was applied for all simulations. For the HREX simulations, 400 million steps were simulated for each replica. The HREX simulation yields the 
$\mathrm{K}_{\mathrm{d}}$ vs. $\beta$ data (Figure 2, main text). For each HREX simulation, the first 50 million steps were discarded prior to data analysis. The optimal values of $\beta$ for the two peptides $\left(\beta_{\mathrm{TAZ1} \text { HIF-1 } \mathrm{opt}}^{\mathrm{ond}}\right.$ $\beta_{\text {TAZ1:CITED2 }}^{\text {opt }}$ that reproduce the experimental $K_{d}$ of the binary complexes were calculated from the $K_{d}$ vs. $\beta$ plot through curve interpolation using equation (4), which describes the relationship between $K_{d}$ and $\beta$ with the entropy change, $\Delta S$, enthalpy change, $\Delta H$, and temperature $T$ as three parameters. The derivation of this equation is shown in a previous paper. ${ }^{11}$ For each unbiased simulation, we simulated 30 billion steps $(0.66 \mathrm{~ms})$ for binary systems and 60 billion steps $(1.32$ ms) for ternary systems with $\beta$ set to $\beta^{\text {opt }}$ calculated from HREX. Snapshots were collected every 10,000 steps for data analysis.

$$
\ln \left(K_{d}\right)=\ln \left(\frac{1660}{V}\right)-\frac{T \Delta S}{R T}+\frac{\Delta H}{R T} \beta-\ln \left\{\exp \left(\frac{T \Delta S}{R T}-\frac{\Delta H}{R T} \beta\right)+1\right\}
$$

Data Analysis. The dissociation constant $\mathrm{K}_{\mathrm{d}}$ is calculated based on the fraction of native contacts (Q) formed between TAZ1 and the ligand. A native contact was considered formed if the pair distance is within $1 \AA$ of the native distance. The ligand is considered to be bound if $Q>0.1$ and the associated $\mathrm{K}_{\mathrm{d}}$ is calculated from the fraction of unbound states $\mathrm{P}_{\mathrm{u}}$ (number of unbound snapshots / total number of snapshots) using equation (5).

$$
K_{d}=\frac{1660}{V} \times \frac{P_{u}^{2}}{1-P_{u}}
$$

Markov State Model Analysis. The unbiased trajectories provide valuable kinetic information of the TAZ1 protein switch and can be better analyzed by a Markov state model ${ }^{14,15}$. As discussed in the main text, we consider CITED2 to be either bound $(\mathrm{Q}>0.1)$ or unbound $(\mathrm{Q}<0.1)$; and HIF$1 \alpha$ to be bound $(\mathrm{Q}>0.3)$, partially bound $(0.1<\mathrm{Q}<0.3)$, or unbound $(\mathrm{Q}<0.1)$. We build a Markov state model using the unbiased trajectory of the CITED2:TAZ1:HIF-1 $\alpha$ ternary complex with 5 
states based on this classification: TAZ1 (free-TAZ1), TAZ1 with bound CITED2 (TAZ1:CITED2 complex), TAZ1 with partially bound HIF-1 $\alpha$ (TAZ1:p-HIF-1 $\alpha$ complex), TAZ1 with bound HIF$1 \alpha$ (TAZ1:HIF-1 $\alpha$ complex), and TAZ1 with bound CITED2 and partially bound HIF-1 $\alpha$ (CITED2:TAZ1:p-HIF-1 $\alpha$ complex), as shown in Figure 3 in the main text. We chose 200 snapshots (2,000,000 dynamic steps) as the lag time for the model as the implied timescale vs. lag time plot (Figure S2) begins to show flattened timescale from 200 timesteps. The Markov state model was built based on the trajectories with discretized states using the MSMBuilder package ${ }^{15}$. To check the Markovianity of the model, we carried out the Chapman-Kolmogorow (CK) test (Figure S3) using the PyEMMA package ${ }^{14}$ and the model prediction and re-estimation are in good agreement. 


\section{SI Figures}

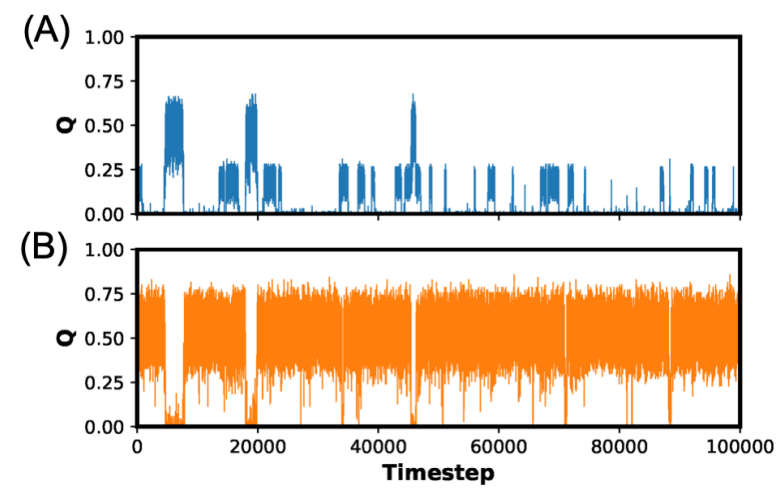

Figure S1. Representative trajectories of fraction of native contacts (Q) of HIF-1 $\alpha$ (A) and CITED2 (B) of the ternary complex with a dielectric constant of 40. HIF-1 $\alpha$ shows two bound states: the bound state $(0.3<\mathrm{Q})$ and the partially bound state $(0.1<\mathrm{Q}<0.3)$. CITED2 shows a single bound state $(\mathrm{Q}>0.1)$.

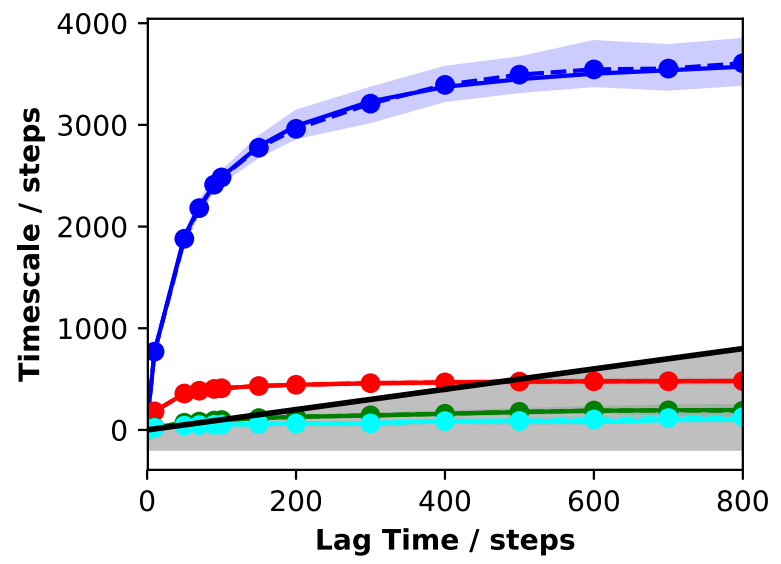

Figure S2. Implied timescales as a function of the lag time of the Markov state model. 

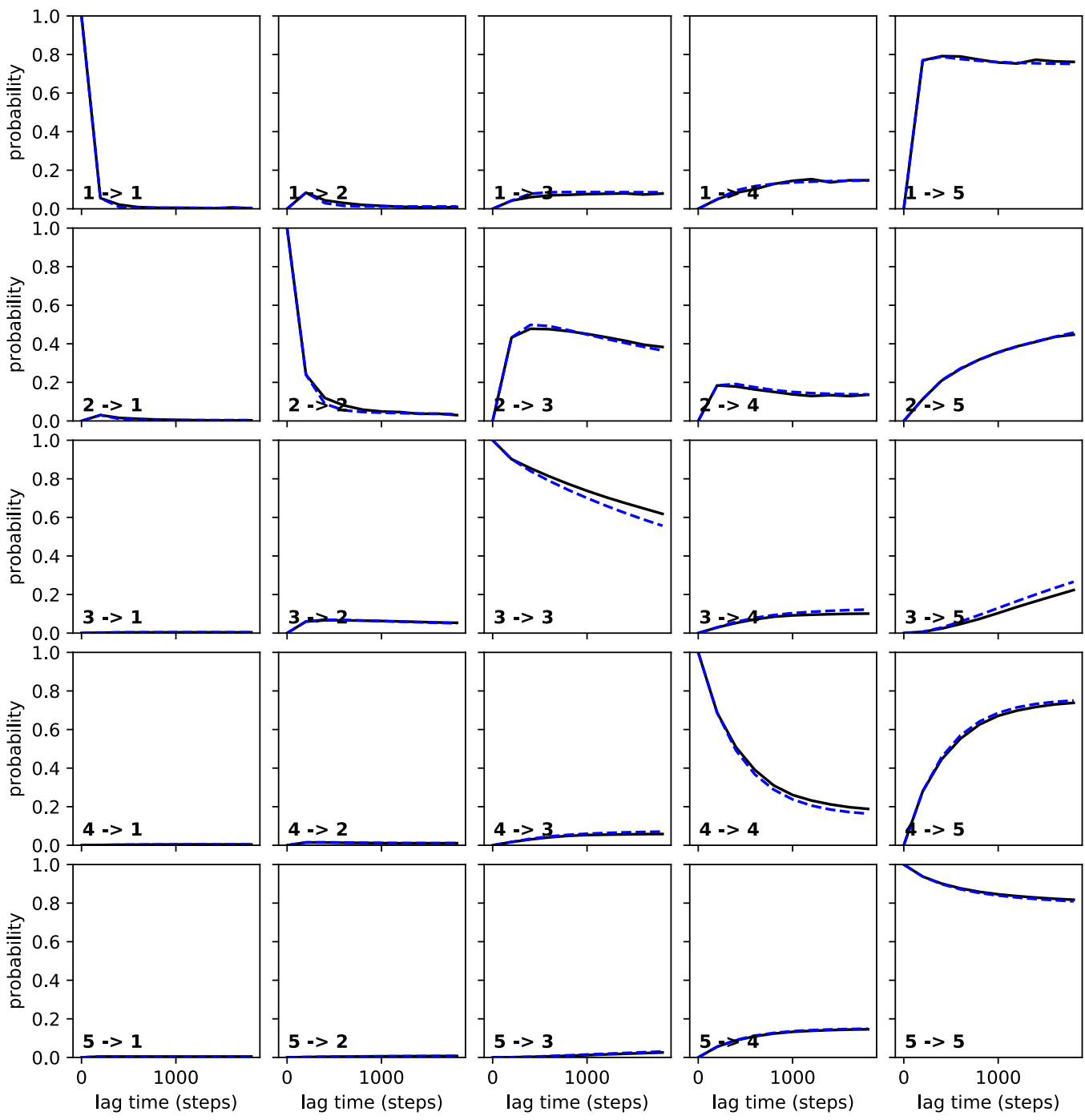

Figure S3. Chapman-Kolmogorow (CK) test of the Markov state model. 


\section{$\begin{array}{lll}\text { (A) TAZ1 } & \text { (B) TAZ1:CITED2 } & \text { (C) TAZ1:HIF-1 } \alpha\end{array}$}

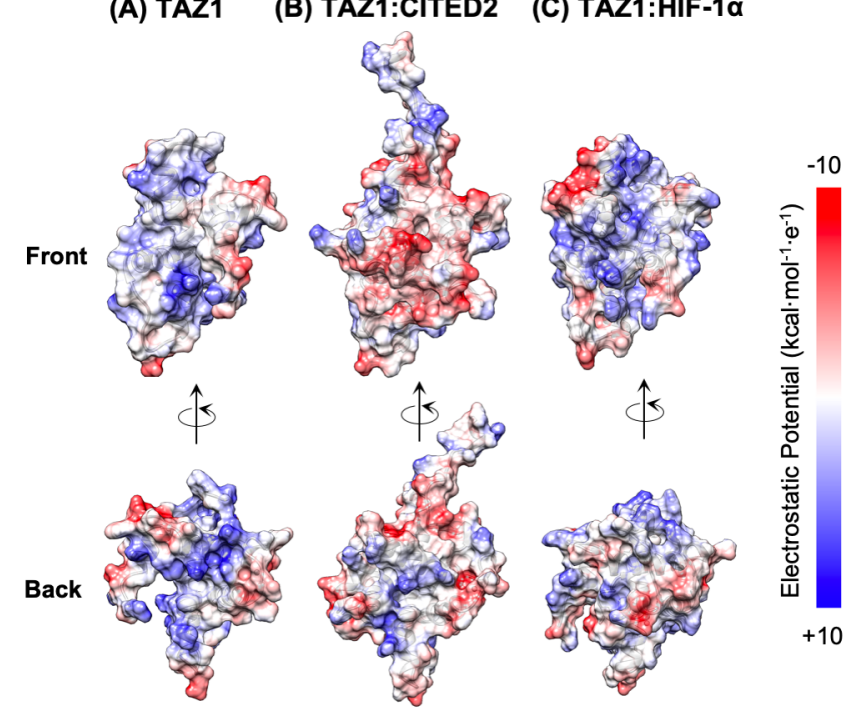

Figure S4. Columbic potential maps of free-TAZ1 (A), TAZ1:CITED2 binary complex (B), and TAZ1:HIF-1 $\alpha$ binary complex (C). 


\section{SI Tables}

Table S1. Kinetic data calculated by unbiased simulations of CITED2:TAZ1 and HIF-1 $\alpha$ binary systems at $\mathrm{D}=40,50,60$ and 80 .

\begin{tabular}{clccc}
\hline Ligand & Bind to & $\mathrm{K}_{\mathrm{d}}(\mathrm{nM})$ & $\mathrm{K}_{\text {on }}\left(\mathrm{M}^{-1} \mathrm{~s}^{-1}\right)$ & $\mathrm{K}_{\text {off }}\left(\mathrm{s}^{-1}\right)$ \\
\hline CITED2 & TAZ1 $(\mathrm{D}=40)$ & 1.20 & $5.34 \times 10^{14}$ & $6.44 \times 10^{6}$ \\
CITED2 & TAZ1 $(\mathrm{D}=50)$ & 1.23 & $9.53 \times 10^{14}$ & $1.17 \times 10^{7}$ \\
CITED2 & TAZ1 $(\mathrm{D}=60)$ & 0.92 & $1.94 \times 10^{14}$ & $1.79 \times 10^{6}$ \\
CITED2 & TAZ1 $(\mathrm{D}=80)$ & 1.04 & $0.47 \times 10^{14}$ & $0.49 \times 10^{5}$ \\
HIF-1 $\alpha$ & TAZ1 $(\mathrm{D}=40)$ & 1.14 & $3.20 \times 10^{14}$ & $3.65 \times 10^{6}$ \\
HIF-1 $\alpha$ & TAZ1 $(\mathrm{D}=50)$ & 1.09 & $9.13 \times 10^{14}$ & $1.00 \times 10^{7}$ \\
HIF-1 $\alpha$ & TAZ1 $(\mathrm{D}=60)$ & 2.50 & $1.01 \times 10^{14}$ & $2.53 \times 10^{6}$ \\
HIF-1 $\alpha$ & TAZ1 $(\mathrm{D}=80)$ & 2.05 & $0.74 \times 10^{14}$ & $1.51 \times 10^{6}$ \\
\hline
\end{tabular}

Table S2. Transition matrix for the 5-state Markov state model using the unbiased trajectories when $\mathrm{D}=40$.

\begin{tabular}{|c|c|c|c|c|c|}
\hline From To & free-TAZ1 & TAZ1:CITED2 & TAZ1:p-HIF-1 $\alpha$ & $\begin{array}{l}\text { CITED2:TAZ1: } \\
\text { p-HIF-1 } \alpha\end{array}$ & TAZ1:HIF- $1 \alpha$ \\
\hline free-TAZ1 & $5.54 \%$ & $76.96 \%$ & $8.38 \%$ & $4.90 \%$ & $4.23 \%$ \\
\hline TAZ1:CITED2 & $0.44 \%$ & $93.78 \%$ & $0.18 \%$ & $5.53 \%$ & $0.08 \%$ \\
\hline TAZ1:p-HIF-1 $\alpha$ & $3.03 \%$ & $11.29 \%$ & $24.00 \%$ & $18.43 \%$ & $43.25 \%$ \\
\hline $\begin{array}{r}\text { CITED2:TAZ1: } \\
\text { p-HIF-1 } \alpha\end{array}$ & $0.14 \%$ & $27.92 \%$ & $1.48 \%$ & $68.77 \%$ & $1.69 \%$ \\
\hline TAZ1:HIF-1 $\alpha$ & $0.21 \%$ & $0.66 \%$ & $6.00 \%$ & $2.93 \%$ & $90.21 \%$ \\
\hline
\end{tabular}


Table S3. Equilibrium population of the 5 states in the CITED2:TAZ1:HIF-1 $\alpha$ ternary complex at different screening lengths when $\mathrm{D}=40$.

\begin{tabular}{rrrrrr}
\hline $\begin{array}{c}\text { Screening } \\
\text { length }\end{array}$ & free-TAZ1 & TAZ1:CITED2 & TAZ1:p-HIF-1 $\alpha$ & $\begin{array}{c}\text { CITED2:TAZ1: } \\
\text { p-HIF-1 } \alpha\end{array}$ & TAZ1:HIF-1 $\alpha$ \\
\hline $1 \AA$ & $81.40 \%$ & $0.88 \%$ & $13.47 \%$ & $0.06 \%$ & $4.18 \%$ \\
$5 \AA$ & $34.80 \%$ & $4.41 \%$ & $28.69 \%$ & $0.98 \%$ & $31.12 \%$ \\
$10 \AA$ & $0.45 \%$ & $74.93 \%$ & $1.20 \%$ & $14.81 \%$ & $8.62 \%$ \\
$15 \AA$ & $0.11 \%$ & $64.66 \%$ & $0.40 \%$ & $34.25 \%$ & $0.58 \%$ \\
$20 \AA$ & $0.05 \%$ & $48.83 \%$ & $0.51 \%$ & $50.29 \%$ & $0.32 \%$ \\
Inf $\AA^{\mathrm{a}}$ & $0.03 \%$ & $14.57 \%$ & $0.69 \%$ & $84.41 \%$ & $0.29 \%$ \\
\hline
\end{tabular}

${ }^{a}$ Infinite screening length.

Table S4. $\mathrm{K}_{\mathrm{d}} \mathrm{S}(\mathrm{M})$ of CITED2 and HIF-1 $\alpha$ in the CITED2:TAZ1:HIF-1 $\alpha$ ternary complex at different screening lengths when $\mathrm{D}=40$.

\begin{tabular}{ccc}
\hline Screening length & $\mathrm{K}_{\mathrm{d}}(\mathrm{CITED} 2) / \mathrm{M}$ & $\mathrm{K}_{\mathrm{d}}(\mathrm{HIF}-1 \alpha) / \mathrm{M}$ \\
\hline $1 \AA$ & $5.25 \times 10^{-2} \pm 1.40 \times 10^{-2}$ & $1.94 \times 10^{-3} \pm 0.44 \times 10^{-3}$ \\
$5 \AA$ & $9.11 \times 10^{-3} \pm 3.73 \times 10^{-3}$ & $1.28 \times 10^{-4} \pm 0.36 \times 10^{-4}$ \\
$10 \AA$ & $8.20 \times 10^{-6} \pm 11.3 \times 10^{-6}$ & $1.24 \times 10^{-3} \pm 0.44 \times 10^{-3}$ \\
$15 \AA$ & $7.79 \times 10^{-8} \pm 13.8 \times 10^{-8}$ & $5.93 \times 10^{-4} \pm 0.90 \times 10^{-4}$ \\
$20 \AA$ & $4.25 \times 10^{-8} \pm 6.36 \times 10^{-8}$ & $2.31 \times 10^{-4} \pm 0.26 \times 10^{-4}$ \\
Inf $\AA^{\mathrm{a}}$ & $6.07 \times 10^{-8} \pm 10.2 \times 10^{-8}$ & $1.23 \times 10^{-5} \pm 0.17 \times 10^{-5}$ \\
\hline
\end{tabular}

${ }^{a}$ Infinite screening length. 


\section{SI References}

(1) Karanicolas, J.; Brooks, C. L., III. The Origins of Asymmetry in the Folding Transition States of Protein L and Protein G. Protein Sci. 2009, 11 (10), 2351-2361.

(2) Hills, R. D.; Brooks, C. L., III. Insights from Coarse-Grained Go Models for Protein Folding and Dynamics. Int. J. Mol. Sci. 2009, 10 (3), 889-905.

(3) Ganguly, D.; Chen, J. Topology-Based Modeling of Intrinsically Disordered Proteins: Balancing Intrinsic Folding and Intermolecular Interactions. Proteins Struct. Funct. Bioinforma. 2011, 79 (4), 1251-1266.

(4) Law, S. M.; Gagnon, J. K.; Mapp, A. K.; Brooks, C. L., III. Prepaying the Entropic Cost for Allosteric Regulation in KIX. Proc. Natl. Acad. Sci. U. S. A. 2014, 111 (33), 1206712072.

(5) Borgia, A.; Borgia, M. B.; Bugge, K.; Kissling, V. M.; Heidarsson, P. O.; Fernandes, C. B.; Sottini, A.; Soranno, A.; Buholzer, K. J.; Nettels, D.; et al. Extreme Disorder in an Ultrahigh-Affinity Protein Complex. Nature 2018, 555 (7694), 61-66.

(6) Miyazawa, S.; Jernigan, R. L. Residue-Residue Potentials with a Favorable Contact Pair Term and an Unfavorable High Packing Density Term, for Simulation and Threading. $J$. Mol. Biol. 1996, 256 (3), 623-644.

(7) Peters, M. B.; Yang, Y.; Wang, B.; Füsti-Molnár, L.; Weaver, M. N.; Merz, K. M. Structural Survey of Zinc-Containing Proteins and Development of the Zinc AMBER Force Field (ZAFF). J. Chem. Theory Comput. 2010, 6 (9), 2935-2947.

(8) Dames, S. A.; Martinez-Yamout, M.; De Guzman, R. N.; Jane Dyson, H.; Wright, P. E. Structural Basis for Hif-1 $\alpha /$ CBP Recognition in the Cellular Hypoxic Response. Proc. Natl. Acad. Sci. U. S. A. 2002, 99 (8), 5271-5276. 
(9) De Guzman, R. N.; Martinez-Yamout, M. A.; Dyson, H. J.; Wright, P. E. Interaction of the TAZ1 Domain of the CREB-Binding Protein with the Activation Domain of CITED2: Regulation by Competition between Intrinsically Unstructured Ligands for Non-Identical Binding Sites. J. Biol. Chem. 2004, 279 (4), 3042-3049.

(10) Berlow, R. B.; Dyson, H. J.; Wright, P. E. Hypersensitive Termination of the Hypoxic Response by a Disordered Protein Switch. Nature 2017, 543 (7645), 447-451.

(11) Wang, Y.; Brooks, C. L., III. Enhanced Sampling Applied to Modeling Allosteric Regulation in Transcription. J. Phys. Chem. Lett. 2019, 10 (19), 5963-5968.

(12) Bussi, G. Hamiltonian Replica Exchange in GROMACS: A Flexible Implementation. Mol. Phys. 2014, 112 (3-4), 379-384.

(13) Eastman, P.; Swails, J.; Chodera, J. D.; McGibbon, R. T.; Zhao, Y.; Beauchamp, K. A.; Wang, L. P.; Simmonett, A. C.; Harrigan, M. P.; Stern, C. D.; et al. OpenMM 7: Rapid Development of High Performance Algorithms for Molecular Dynamics. PLoS Comput. Biol. 2017, 13 (7), e1005659.

(14) Scherer, M. K.; Trendelkamp-Schroer, B.; Paul, F.; Pérez-Hernández, G.; Hoffmann, M.; Plattner, N.; Wehmeyer, C.; Prinz, J. H.; Noé, F. PyEMMA 2: A Software Package for Estimation, Validation, and Analysis of Markov Models. J. Chem. Theory Comput. 2015, $11(11), 5525-5542$.

(15) Harrigan, M. P.; Sultan, M. M.; Hernández, C. X.; Husic, B. E.; Eastman, P.; Schwantes, C. R.; Beauchamp, K. A.; McGibbon, R. T.; Pande, V. S. MSMBuilder: Statistical Models for Biomolecular Dynamics. Biophys. J. 2017, 112 (1), 10-15. 